\title{
Cardinal Invariants for Commutative Group Algebras
}

\author{
Peter DancheV
}

\begin{abstract}
A new kind of major structural invariants for commutative group algebras and pairs of commutative group algebras are here obtained. The present statements are a sequel to our recent results published in Ricerche Math. (Napoli, 2001 and 2003) plus Rend. Circolo Mat. Palermo (2002).
\end{abstract}

\section{INTRODUCTION}

This paper is a natural supplement to previous results in this aspect due to the author [3-9]. It is to be understood throughout that all groups considered in the current work are Abelian. Following the notions from [12], if among the pure subgroups of a group $G$ which contain $A$ there exists a minimal one, we say that $A$ is contained in, or is imbedded in, a minimal pure subgroup of $G$. We emphasize that the subgroup $A$ of $G$ is said to be purifiable if, among the pure subgroups of $G$ containing $A$, there is a minimal one. Such a minimal pure subgroup of $G$ is called a pure hull of $A$ in $G$. The terminology, notations and other material on Abelian groups not expressly introduced here follow the usage of [10] and [7]. For $F$ an arbitrary field of $\operatorname{char} F=p, F G$ will denote the group algebra of $G$ over $F$. For an arbitrary subgroup $A$ in $G,(F G, F A)$ designates a pair of $F$-group algebras. Recall that $V(R G)$ is the normalized group of units with $p$-component $S(R G)$, and $I(R G ; A)$ denotes the relative augmentation ideal of $R G$ with respect to $A$, whenever $R$ is a commutative ring with identity. For the basic background on group rings see [15] and [16].

In the theory of commutative group algebras a central problem is that of deducing information about $G$ from the $F$-group algebra $F G$ as well as about the group pair $(G, A)$ from the $F$-pair $(F G, F A)$. The principal known results in this direction may be found in [16], [2], [15], [3-9]. Moreover, of some importance are also the following other invariants of $G$ and $(G, A)$, which are in the focus of our interest, namely:

2000 Mathematics Subject Classification. Primary: 20C07; Secondary: 16S34.

Key words and phrases. Group algebras, purifiable subgroups, pure hulls, defects, covering dimensions, complemental dimensions, Hill's numbers. 
(a) For every ordinal $\alpha$, the $\alpha$-th defect of $A$ in $G$ is the vector space over the field $F_{p}$ of $p$-elements (see [17])

$$
D_{\alpha}(G, A)=(G / A)^{p^{\alpha}}[p] /\left(G^{p^{\alpha}}[p] A\right) / A \text {. }
$$

These invariants play a key role in the intersection problem, and shed an information on the purity and isotypity as well.

(b) Let $A$ be purifiable in $G$. For every integer $n \geq 0$, we define the dimension of $(P / A)^{p^{n}}[p]$ for the pure hull $P$ of $A$ in $G$ as a vector $F_{p^{-}}$space by (cf. $[17])$

$$
\operatorname{Cov}_{n}(G, A)=\operatorname{dim}_{F_{p}}(P / A)^{p^{n}}[p] .
$$

This cardinal number is called the $n$-th covering dimension of $A$ in $G$. It is a relative invariant of $A$ in $G$. We also set $\operatorname{Def}(A)=\operatorname{dim}_{F_{p}}(N / A)[p]$, where $N$ is a neat hull of $A$ in $G$ and $A$ is neatable in $G$. If $N$ is pure in $G$, then $N$ is vertical in $G \Rightarrow \operatorname{Def}(A)=\operatorname{Cov}_{0}(G, A)$.

(c) Let $A$ be purifiable in $G$. For each natural $n \geq 0$, we put the dimension of $G^{p^{n}}[p] / P^{p^{n}}[p]$ by (see $\left.[17]\right)$

$$
\operatorname{Com}_{n}(G, A)=\operatorname{dim}_{F_{p}}\left(G^{p^{n}}[p] / P^{p^{n}}[p]\right) .
$$

The last cardinal number is called the $n$-th complemental dimension of $A$ in $G$. It is a relative invariant of $A$ in $G$. Besides, since $P$ is pure in $G$, we trivially detect that $G^{p^{n}}[p] / P^{p^{n}}[p] \cong(G / P)^{p^{n}}[p]$, and therefore

$$
\operatorname{Com}_{n}(G, A)=\operatorname{dim}_{F_{p}}(G / P)^{p^{n}}[p] .
$$

(d) P. Hill in [11] has introduced the following cardinal functions (called Hill numbers or Hill invariants): For $\mu$ a limit ordinal not cofinal with $\omega=\omega_{0}$, set

$$
E_{\mu}=\bigcap_{\substack{\lambda<\mu, \lambda+\sigma=\mu}}\left(G^{p^{\lambda}} A / A\right)^{p^{\sigma}} /\left(G^{p^{\mu}} A / A\right)
$$

Then

$$
H_{\mu}(\lambda)= \begin{cases}\operatorname{dim}\left(G^{p^{\alpha}}[p] / G^{p^{\alpha+1}}[p]\right), & \text { if } \mu=0 \text { and } \alpha<\infty \\ \operatorname{dim}\left(E_{\mu}^{p^{\alpha}}[p] / E_{\mu}^{p^{\alpha+1}}[p]\right), & \text { if } \mu \neq 0 \text { and } \alpha<\infty \\ \operatorname{dim} E_{\mu}^{p^{\alpha}}[p], & \text { if } \mu \neq 0 \text { and } \alpha=\infty .\end{cases}
$$

(e) We select the relative $p$-Warfield invariants of $A$ in $G$ with respect to the ordinal $\alpha$ as follows

$$
W_{\alpha, p}(G, A)=\operatorname{dim}_{F_{p}}\left(G^{p^{\alpha}} /\left(\left[\left(G^{p^{\alpha+1}} A\right) \cap G^{p^{\alpha}}\right]\left(G^{p^{\alpha}}\right)_{t}\right)\right) .
$$

This construction strengthens the classical long-known definition of the ordinary Warfield $p$-invariants.

We continue with the statement of the major assertions. 


\section{Main Results}

Now we are in position to formulate and prove the attainments on functional invariants for abelian group algebras, motivated this article. Some of them were previously announced in [9]. And so, we start with

Theorem 1 (Invariants). The following claims are valid:

(*) For any ordinal $\alpha, W_{\alpha, p}(G, A)$ is an isomorphic cardinal invariant of $(F G, F A)$.

(**) For each ordinal $\alpha, D_{\alpha}(G, A)$ and $H_{\mu}(\alpha)$ are structural cardinal invariants for $(F G, F A)$.

(***) For every purifiable subgroup $A$ of $p$-primary $G, \operatorname{Cov}_{n}(G, A)$ and $\operatorname{Com}_{n}(G, A)$ are functional cardinal invariants of $(F G, F A)$.

Begin further with a statement consequence.

Proposition 1 (Properties). Suppose $(F G, F A) \cong(F H, F B)$ as pair of F-algebras. Then the following hold:

$\left.{ }^{\circ}\right)$ If $A$ is pure (isotype) in $G_{p}$, then $B$ is pure (isotype) in $H_{p}$.

$\left(^{\circ}\right)$ If $A$ is purifiable in $G_{p}$, then $B$ is purifiable in $H_{p}$.

${ }^{(\infty)}$ ) If $A$ is an intersection of pure (isotype) subgroups in $G_{p}$, then $B$ is an intersection of pure (isotype) subgroups in $H_{p}$.

We can now attack their proofs, which are demonstrated in the next paragraph.

Proofs of Preliminary and Central Affirmations. First and foremost we list (cf. $[3,4,6])$ a lemma needed for our presentation, namely:

Lemma 1. Let $T \leq A \leq G$ and $M \leq G$. Then

$$
I(F G ; A M)=I(F G ; A)+I(F G ; M) .
$$

Besides for $1 \in P \leq R$, the following intersection ratio holds true

$$
I(F A ; T) \cap P M=I(P(A \cap M) ; T \cap M) .
$$

Now, we are ready to begin with the proofs. In fact, we proceed PROOF of: $(*)$

Since

$$
\left(G^{p^{\alpha}}\right)_{t}=\left(G_{t}\right)^{p^{\alpha}}=\left(G_{p}\right)^{p^{\alpha}}\left(\coprod_{q \neq p} G_{q}\right)
$$

we observe that

$$
\left[\left(G^{p^{\alpha+1}} A\right) \cap G^{p^{\alpha}}\right]\left(G^{p^{\alpha}}\right)_{t}=\left[\left(G^{p^{\alpha+1}} A\right) \cap G^{p^{\alpha}}\right]\left(G^{p^{\alpha}}\right)_{p} .
$$

Henceforth, we apply the methods from $[3,4,6]$ together with the Lemma to conclude that the fundamental ideals $I\left(F G ; G_{p}^{p^{\alpha}}\right)$ along with

$$
I\left(F G ; G^{p^{\alpha+1}} A\right)=I\left(F G ; G^{p^{\alpha+1}}\right)+I(F G ; A) \quad \text { and } \quad I\left(F G ;\left(G^{p^{\alpha+1}} A\right) \cap G^{p^{\alpha}}\right)
$$


may be recovered by $(F G, F A)$. As a finish, exploiting a result due to Karpilovsky [15], we have that the explored relative $p$-invariants of Warfield can be recaptured from the $F$-pair $(F G, F A)$, as wanted.

PROOF of: $(* *)$

The fact that $D_{\alpha}(G, A)$ is an invariant of $(F G, F A)$ follows thus. As we have seen in $[3,4], I\left(F G ; G^{p^{\alpha}}[p]\right)$ can be retrieved from $F G$. On the other hand

$$
I\left(F G ; G^{p^{\alpha}}[p] A\right)=I\left(F G ; G^{p^{\alpha}}[p]\right)+I(F G ; A)=I\left(F G ; G^{p^{\alpha}}[p]\right)+F G \cdot I(F A ; A)
$$

may be obtained from $(F G, F A)$ using the Lemma. Furthermore by [15]

$$
\begin{aligned}
& \operatorname{dim}_{F_{p}}(G / A)^{p^{\alpha}}[p] /\left(G^{p^{\alpha}}[p] A\right) / A=\operatorname{dim}_{F}\left(I\left(F(G / A) ;(G / A)^{p^{\alpha}}[p]\right) /\right. \\
& \left.\quad /\left(I(F(G / A) ; G / A) \cdot I\left(F(G / A) ;(G / A)^{p^{\alpha}}[p]\right)+I\left(F(G / A) ; G^{p^{\alpha}}[p] A / A\right)\right)\right) .
\end{aligned}
$$

Since $F(G / A) \cong F G / I(F G ; A)=F G / F G \cdot I(F A ; A)$ may be gotten by $(F G, F A)$ and moreover

$$
I\left(F G ; G^{p^{\alpha}}[p] A\right) / I(F G ; A) \cong I\left(F(G / A) ; G^{p^{\alpha}}[p] A / A\right)
$$

can be determined also from this pair, the result holds directly by virtue of $[3,4]$ or $[15]$.

Now, we shall apply the same procedure to get that $H_{\mu}(\alpha)$ are invariants for the pair $(F G, F A)$. For this purpose it is enough to establish only that $I\left(F\left(G / A / G^{p^{\mu}} A / A\right) ; E_{\mu}^{p^{\tau}}[p]\right)$ is an invariant of $(F G, F A)$, i.e in other words it is sufficient to verify via $[3,4]$ and [15] that $I\left(F E_{\mu} ; E_{\mu}\right)$ can be recovered from $(F G, F A)$. Indeed, we consider the $F$-algebra $F E_{\mu}$. Evidently

$$
F E_{\mu}=\bigcap_{\substack{\lambda<\mu \\ \lambda+\sigma=\mu}} F\left[\left(G^{p^{\lambda}} A / A\right)^{p^{\sigma}} /\left(G^{p^{\mu}} A / A\right)\right] .
$$

After this, we shall check that $F\left[\left(G^{p^{\lambda}} A / A\right)^{p^{\sigma}} /\left(G^{p^{\mu}} A / A\right)\right]$ may be determined by $(F G, F A)$. Indeed, this follows from noticing that the factor-algebra is isomorphic to

$$
F\left[\left(G^{p^{\lambda}} A / A\right)^{p^{\sigma}}\right] / I\left(F\left(G^{p^{\lambda}} A / A\right)^{p^{\sigma}} ;\left(G^{p^{\mu}} A / A\right)\right) .
$$

But,

$$
F\left(G^{p^{\lambda}} A / A\right)^{p^{\sigma}}=\left[F\left(G^{p^{\lambda}} A / A\right)\right]^{p^{\sigma}},
$$

and

$$
F\left(G^{p^{\lambda}} A / A\right) \cong F\left(G^{p^{\lambda}} A\right) / I\left(F\left(G^{p^{\lambda}} A\right) ; A\right)
$$

where

$$
F\left(G^{p^{\lambda}} A\right)=F G^{p^{\lambda}} \cdot F A=(F G)^{p^{\lambda}} \cdot F A
$$

and

$$
I\left(F\left(G^{p^{\lambda}} A\right) ; A\right)=F\left(G^{p^{\lambda}} A\right) \cdot I(F A ; A) .
$$

On the other hand

$$
F\left(G^{p^{\mu}} A / A\right) \cong F\left(G^{p^{\mu}} A\right) / I\left(F\left(G^{p^{\mu}} A\right) ; A\right),
$$


where as above

$$
F\left(G^{p^{\mu}} A\right)=F G^{p^{\mu}} \cdot F A \text { and } I\left(F\left(G^{p^{\mu}} A\right) ; A\right)=F G^{p^{\mu}} \cdot I(F A ; A) .
$$

So, our claim is substantiated.

Proof of: $(* * *)$

Since $F G=F H, F A=F B$ and $F P=F M$ for some pure hulls $P$ of $A$ in $G$ and $M$ of $B$ in $H$ respectively (see the constructions below), we detect that the algebras $F(P / A)$ and $F(G / P)$ can be extracted from $(F G, F A)$ and $(F H, F B)$.

The theorem is proved in general after all.

We now concentrate on the verification of the corollary.

$\left.{ }^{\circ}\right)$ Since $A$ is isotype in $G$, we deduce $V(F B)=V(F A)$ is $p$-isotype in $V(F G)=V(F H)$. Thereby, $B$ as $p$-isotype in $V(F B)$ must be $p$-isotype in $V(F H)$ whence it is isotype in $H_{p}$.

We give an independent approach to confirm once again $\left(^{\circ}\right)$. Exploiting [17] and [18], $A$ is balanced (nice and isotype) in $G_{p}$ if and only if $D_{\alpha}(G, A)=0$ for each ordinal $\alpha$. But, as we have argued in the Theorem, $D_{\alpha}(G, A)$ can be gotten from $(F G, F A)$. Besides, $A$ is pure in $G_{p}$ if and only if $D_{n}(G, A)=0$ for all naturals $n$.

( $\left.{ }^{\circ}\right)$ Assume $A \subseteq P$ where $P$ is a minimal pure subgroup of $G_{p}$, i.e. $P$ is a pure hull of $A$ in $G_{p}$; in other words there is no proper subgroup of $P$ that is pure in $G_{p}$. After this, we may presume that $F$ is perfect. By hypothesis, $F G=F H$ and $F A=F B$ for some subgroup $B \leq H_{p}$. Given $B \subseteq M \subseteq H_{p}$ so that $M$ is pure in $H_{p}$. We search such a minimal group $M$ with this property. Since $A \subseteq S(F A)=S(F B) \subseteq S(F M)$ and since $S(F M)$ is pure in $S(F H)=S(F G)$, it follows at once that $P \subseteq S(F M)$. Henceforth, we choose $M \leq H_{p}$ on which $F M=F P$. Furthermore,

$$
\begin{aligned}
M \cap H_{p}^{p^{n}} & \subseteq S(F M) \cap S^{p^{n}}(F H)=S(F P) \cap S^{p^{n}}(F G)= \\
& =S(F P) \cap S\left(F G^{p^{n}}\right)=S\left(F\left(P \cap G^{p^{n}}\right)\right)= \\
& =S\left(F P^{p^{n}}\right)=S^{p^{n}}(F P)=S^{p^{n}}(F M),
\end{aligned}
$$

hence

$$
M \cap H_{p}^{p^{n}} \subseteq S^{p^{n}}(F M) \cap M=S\left(F M^{p^{n}}\right) \cap M=M^{p^{n}},
$$

for each natural number $n$, that is $M$ is pure in $H_{p}$. Next, if there is $N \subset M$ such that $N$ is pure in $H_{p}$, we select $T \leq G_{p}$ with $F N=F T$. As above, we may infer that $T$ is pure in $G_{p}$. Moreover, $T \subseteq F N \subset F M=$ $F P$ whence $T \subset P$, because if $T=P$ we have that $F M=F N$ jointly with $N \subset M$ force $M=N$, which is the desired contradiction. Thereby, $M$ is a minimal pure subgroup of $H_{p}$ containing $B$. So, $M$ is a pure hull of $B$ in $H_{p}$ and consequently $B$ is purifiable in $H_{p}$, as expected.

$\left({ }^{\circ}\right)$ Utilizing [17], for each ordinal number $\alpha,\left(G^{p^{\alpha}}[p] A\right) / A=1$ yields $(G / A)^{p^{\alpha}}[p]=1$. But, owing to our method described above, the two 
factor-groups may be retrieved from the couple $(F G, F A)$. So, again invoking to [17], the proof of this point is fulfilled.

The proof of the corollary is completed.

Claim 1. Assume $P \leq G_{p}$. Then $P$ is minimal pure in $G_{p} \Leftrightarrow P$ is minimal pure in $S(F G)$.

Proof. If there exists a pure subgroup $K$ of $S(F G)$ so that $K \subset P$, we obtain that $K$ must be pure in $G_{p}$ which contradicts the minimality of $P$ in $G_{p}$.

Conversely, if $L$ is a pure subgroup of $G_{p}$ and is contained in $P$, the purity of $G_{p}$ in $S(F G)$ and its transitivity imply that $L$ is pure in $S(F G)$. But this fails owing to the minimality of $P$ in $S(F G)$.

Corollary 1. Assume $A \leq G_{p}$. Then $A$ is purifiable in $G_{p} \Leftrightarrow A$ is purifiable in $S(F G)$.

We end the investigation with

Problems. What are the divisible hull and the pure hull for the group $S(F G)$ ?

In [4] we have asked whether or not $F G$ determines $G / B_{u}$, where $B_{u}$ is an upper basic subgroup of $G$. We now precise this as turn our attention to the question for the existence of invariance of $I\left(F G ; B_{u}\right)$ from $F G$. In that aspect, does it follow that $F G=F H$ implies

$$
F\left(G / H^{\left(G_{p}\right)}\right) \cong F\left(H / H^{\left(H_{p}\right)}\right) \quad \text { and } \quad I\left(F G ; H^{\left(G_{p}\right)}\right)=I\left(F H ; H^{\left(H_{p}\right)}\right)
$$

whenever $H^{\left(G_{p}\right)}$ and $H^{\left(H_{p}\right)}$ are $G_{p}$-high and $H_{p}$-high subgroups of $G$ and $H$, respectively. For the convenience of the reader, we emphasize that a subgroup $K$ of $G$ is $G_{p}$-high if it is maximal with respect to $\cap G_{p}=1$, that is $K[p]=1$ and $K$ is pure in $G$ (see, for instance, [13] or [14]). Thus if $F G \cong F H$ and $G$ being $p$-splitting $\left(G_{p}\right.$ is a direct factor of $\left.G\right)$ yield that $H$ is $p$-splitting, then $F G \cong F H$ assures $F G_{p} \cong F H_{p}$.

Let $\mathbb{N}$ be the set of nonnegative integers, and let $B=\oplus_{i \in I}\left\langle b_{i}\right\rangle$ be the direct sum of cyclic groups with order $\left(b_{i}\right)=p^{i+1}$. Denote by $B^{-}$the torsion-completion of $B$. If $G$ is a pure subgroup of $B^{-}$, let

$$
I(G)=\left\{i \in \mathbb{N} \mid i^{t h} \text { Ulm invariant of } G \text { is nonzero }\right\} .
$$

Beaumont and Pierce introduced a further invariant for $G$, archived in [1] (see [19] too), namely

$$
U(G)=\{I(A) \mid A \text { is a pure torsion-complete subgroup of } G\} .
$$

Clearly, $U(G)$ is a (boolean) ideal in $P(\mathbb{N})$, the power set of $\mathbb{N}$.

A problem of central interest is whether $U(G)$ is isomorphically retrieved from the $F$-algebra $F G$.

However, these are a problem of some other study.

Acknowledgement. The author's thanks are due to the referee for the valuable comments and suggestions. 


\section{REFERENCES}

[1] R.A. Beaumont and R. S. Pierce, Some invariants of p-groups, Michigan Math. J., 11 (1964), 137-149.

[2] D. Beers, F. Richman and E. Walker, Group algebras of abelian groups, Rend. Sem. Mat. Univ. Padova, 69 (1983), 41-50.

[3] P. Danchev, Numerical invariants for commutative group algebras, Ricerche di Mat., (2)50 (2001), 323-336.

[4] P. Danchev, Invariants for group algebras of abelian groups, Rend. Circolo Mat. Palermo, (3)51 (2002), 391-402.

[5] P. Danchev, A new simple proof of the W. May's claim: $F G$ determines $G / G_{0}$, Rivista Mat. Univ. Parma, 1 (2002), 69-71.

[6] P. Danchev, Numerical invariants for modular group algebras of abelian groups, Ricerche di Mat., (2)52 (2003), 197-202.

[7] P. Danchev, Isomorphism of commutative group algebras over all fields, Rend. Istit. Mat. Univ. Trieste, 35 (2003), 1-18.

[8] P. Danchev, Honest subgroups in commutative group rings, Rivista Mat. Univ. Parma, 3 (2004), 19-23.

[9] P. Danchev, Numerical invariants for group algebras of abelian groups, Compt. Rend. Acad. Bulg. Sci., (11)57 (2004), 5-6.

[10] L. Fuchs, Infinite Abelian Groups, I and II, Mir, Moscow, 1974 and 1977 (In Russian).

[11] P. Hill, On the structure of abelian p-groups, Trans. Amer. Math. Soc., 288 (1985), 505-525.

[12] P. Hill and C. Megibben, Minimal pure subgroups in primary groups, Bull. Soc. Math. France, 92 (1964), 251-257.

[13] J. Irwin, High subgroups of abelian torsion groups, Pac. J. of Math., 11 (1961), 1375-1384.

[14] J. Irwin and E. Walker, On N-high subgroups of abelian groups, Pac. J. of Math., 11 (1961), $1363-1374$.

[15] G. Karpilovsky, Unit Groups of Group Rings, North-Holland, Amsterdam, 1989.

[16] W. May, Commutative group algebras, Trans. Amer. Math. Soc., (1)136 (1969), 139-149.

[17] T. Okuyama, On purifiable subgroups and the intersection problem, Pac. J. of Math., (2)157 (1993), 311-324.

[18] T. Okuyama, On purifiable subgroups in arbitrary abelian groups, Comm. in Algebra, (1)28 (2000), 121-139.

[19] J. Werth Jr., Maximal pure subgroups of torsion complete abelian p-groups, Pac. J. of Math., (1)50 (1974), 307-316.

13, General Kutuzov Street

Block 7, Floor 2, Apartment 4

4003 PLOVDIV

BULGARIA

E-mail address: pvdanchev@yahoo.com 